\title{
Rol del psicólogo en establecimientos particulares pagados del Gran Concepción, Chile: Un proceso de co-construcción
}

\author{
Role of psychologists who work in private school in Concepción, Chile: \\ A co-construction process
}

Papel dos psicólogos que trabalham em escolas privadas, em Concepción, Chile: Um processo de co-construção

\section{Constanza García Costa, ${ }^{\text {a } G l o r i a ~ C a r r a s c o ~ S a n h u e z a, ~}{ }^{\mathrm{a}}$ Mónica Mendoza Barra, ${ }^{\mathrm{a}}$ Cristhian Pérez Villalobos ${ }^{\mathrm{b}}$}

${ }^{\text {a} F a c u l t a d ~ d e ~ P s i c o l o g i ́ a, ~ U n i v e r s i d a d ~ d e l ~ D e s a r r o l l o . ~ A i n a v i l l o ~ 456, ~ C o n c e p c i o ́ n, ~ C h i l e . ~ T e l f .: ~ 56-41-2268773 . ~}$ Correo electrónico: cgarciac@udd.cl,gcarrascos@udd.cl, mmendoza@udd.cl

bDepartamento de Educación Médica, Facultad de Medicina, Universidad de Concepción. Chacabuco esquina Janequeo, 5to piso, Concepción, Chile. Telf.: 56-41-2202932. Correo electrónico: cperezv@udec.cl

\section{RESUMEN}

Existen múltiples visiones sobre las funciones del psicólogo educacional e importantes discrepancias entre la propuesta teórica y las expectativas reales del sistema. Ante esto, la presente investigación analiza el rol del psicólogo en establecimientos particulares pagados del Gran Concepción según la perspectiva de sus actores. Este estudio, cualitativo y desde la teoría fundamentada, realizó entrevistas semi-estructuradas a 14 participantes incluyendo psicólogos, profesores, directivos, alumnos y apoderados de estos establecimientos. Se exhiben los resultados relacionales de las codificaciones axial y selectiva. A nivel de resultados, destaca la importancia del contexto y particularidad de cada centro educativo en el rol del psicólogo, el cual se va co-construyendo entre los actores que se desenvuelven en él, y según las necesidades y recursos con que cuentan.

Palabras clave: psicólogo educacional, psicología educacional, gestión escolar.

\begin{abstract}
There are several perspectives concerning the role of the educational psychologist which reveal deep differences between universities and schools expectancies about it. The aim of the present research is to analyze the role of psychologists in private schools in Concepción, Chile, according to participants' experiences. In this study, following qualitative and Grounded Theory principles, 14 individuals were interviewed including psychologists, teachers, directives, students and parents. Outcomes from axial and selective coding are showed. Results highlight relevance of each educative center particularities for role assumed by psychologists, which is co-constructed between different actors, depending on their needs and the resources available.
\end{abstract}

Key words: educational psychologist, educational psychology, school management.

\section{RESUMO}

Existem várias perspectivas sobre o papel do psicólogo educacional que revelam profundas diferenças entre as expectativas do sistema escolar e da universidade sobre o assunto. $\mathrm{O}$ objetivo da presente pesquisa é analisar o papel do psicólogo na escolas privadas, em Concepción, Chile, de acordo com seus atores principais. Neste estudo, seguindo os princípios da pesquisa qualitativa e Grounded Theory, foram entrevistados 14 agentes, incluindo psicólogos, professores, directivas, alunos e pais. $\mathrm{O}$ artigo presenta os resultados da codificação axial e seletiva. Os resultados reforçam a importância das particularidades de cada centro educativo para o papel assumido pelo psicólogo, que é co-construído entre as diferentes partes interessadas, dependendo de suas necessidades e recursos disponíveis.

Palavras chave: psicólogo educacional, psicologia educacional, administração escolar. 


\section{INTRODUCCIÓN}

\subsection{LA PSICOLOGÍA EDUCACIONAL EN TIEMPOS DE REFORMA}

La Reforma Educacional comienza a implementarse el año 1994, atendiendo a dos motivos principales: por una parte, los problemas de calidad y equidad de la educación y, por otra, los requerimientos sociales que la educación no lograba satisfacer (Raczynski \& Muñoz, 2007). Sin embargo, desde esa fecha, la Reforma no ha logrado cubrir todos los objetivos inicialmente propuestos.

Como señalan Raczynski \& Muñoz (2007), el movimiento estudiantil de fines del primer semestre del año 2006 dejó en evidencia uno de los problemas más desafiantes de nuestro país: la educación no ha logrado abrir oportunidades de desarrollo equitativo. La segmentación social del servicio educativo y la baja calidad de la enseñanza son razones fundamentales por las cuales nuestra educación no es todavía el verdadero motor del desarrollo. Esto se refleja, por ejemplo, en los resultados de los establecimientos municipalizados que se encuentra muy por debajo del promedio nacional de 250 puntos, mientras que los establecimientos particulares muestran un promedio cercano a los 300 puntos, situándose una desviación estándar por sobre el promedio del país (Santander, 2006). En esta línea, la OPECH (2006) señala que la calidad en educación parece estar reservada para quienes puedan pagar por ella.

Sin embargo, Eyzaguirre y Le Foulon (2001) plantean que a nivel internacional, toda nuestra población, incluidos los sectores con mayores recursos, tienen un rendimiento inferior a los países que presentan buenos niveles educacionales. Como estos mismos autores señalan, Chile se ubica en los últimos lugares entre las 38 naciones evaluadas por la prueba TIMSS y las 14 evaluadas por el IALS (Estudio Internacional del Nivel Lector de Adultos).

Sumado a estos malos resultados académicos, la Reforma Educacional exige a los establecimientos educacionales dar respuesta a problemas que antes eran considerados responsabilidad de la familia, debiendo hacerse cargo de las acciones para la resolución de la crisis valórica de la sociedad actual, y asumiendo la formación de los alumnos en áreas afectivas y sociales. Esto, a su vez, conlleva la exigencia a los profesores de ser expertos en temas que van más allá de su disciplina, requiriendo de un apoyo para cumplir esta labor (Banz Liendo, 2002).

Una de las disciplinas que podría estar al servicio de estos nuevos desafíos educativos es justamente la Psicología Educacional, área de especialidad cuya base conceptual se arraiga en la psicología y la educación (American Psychologycal Association, APA, 2005), y que estudia los procesos de enseñanza y aprendizaje y el contexto en el que se producen, aplicando los métodos y las teorías de la psicología (Arancibia, Herrera \& Strasser, 1997; Woolfolk, 2006).

Si bien, de acuerdo a Forns (1994), desde los inicios de la psicología se ha reconocido su papel en el contexto educativo como apoyo a la mejora de la calidad educativa, la Psicología Educativa ha ido cambiando en el tiempo, pudiendo distinguirse cuatro fases de desarrollo: 1) Una primera etapa fuertemente ligada a la educación especial, centrándose en las diferencias individuales, diagnóstico y tratamiento de niños con problemas; 2) una segunda etapa en donde se amplía el espectro de atención a los aspectos emocionales, afectivos y sociales de todos los alumnos; 3) una tercera fase en que el 
psicólogo comienza a apoyar la práctica docente, convirtiéndose en un puente entre el conocimiento psicológico y la práctica escolar; y, por último, 4) la fase actual, en que se dejan de considerar los conflictos como fenómenos individuales, dándose gran relevancia al contexto en que éstos se desarrollan (Colodrón, 2004). Esta última etapa hace que en la actualidad el psicólogo educacional deba realizar su actividad profesional dentro de un sistema dinámico, enfocando su quehacer a todo el sistema si es preciso y no sólo a una parte del mismo, como puede ser el alumno (Barca, 1998).

En la actualidad, la psicología educativa se ha constituido en una disciplina multiparadigmática que adquiere una gran relevancia y, a la vez, se convierte en una necesidad para las instituciones educadoras, en especial las escuelas, los colegios y las universidades, en donde intervienen, además, otros actores de la comunidad educativa como son padres de familia, docentes y directivos, todos ellos implicados en la mejora del proceso educativo (Hernández, 2008). Pese a esto, en Chile no existe ninguna normativa legal ni institucional que regule el desempeño del psicólogo en el ámbito educativo (Redondo, 2007).

\subsection{ROL DEL PSICÓLOGO}

En línea con lo anterior, Mena (1992) describe al psicólogo educacional como el psicólogo de la unidad o sistema educativo y no sólo de los alumnos, cuya labor sería apoyar y asesorar para que el sistema logre sus fines. Para esto, el psicólogo debe conjugar las distintas perspectivas y conocimientos acerca del proceso de enseñanza-aprendizaje y colaborar en la construcción de nuevas alternativas de solución. Según Banz Liendo (2002), el psicólogo debe poseer una concepción de la escuela como una organización que aprende, por lo que su labor debe ser la de promotor y colaborador del cambio que en esta se produce, más que de hacedor directo (Selvini \& Cirrillo, 1997). De esta forma, debe entender que no es requerido para resolver problemas, sino para participar en la co-construcción de intervenciones en las que aporta su mirada psicológica (Banz Liendo, 2002).

Para esto, su procedimiento general de intervención en la escuela debe implicar una evaluación y diagnóstico de la situación educativa, intervención, seguimiento y evaluación final (Campos, 1995). Esto, pues debe ser capaz de evaluar el estado actual de la institución y contribuir en definir hasta donde ésta puede llegar con su apoyo (Banz Liendo, 2002), y asimismo, debe reconocer y atender a las particularidades de la organización educativa y del contexto en que trabaja, ya que cualquier herramienta que se aplique desde la psicología puede ser insuficiente si no se considera el contexto específico (Diversidad Educativa, 2007).

Ahora, en términos de los campos de acción del psicólogo, éstos incluyen los ámbitos personales, familiares, organizacionales, institucionales, socio-comunitarios y educativos en general del establecimiento en su conjunto, y sus potenciales clientes son todos aquellos actores que en él actúan y viven, es decir, directivos, docentes, alumnos y apoderados (Campos, 1995; Selvini \& Cirillo, 1997). Tal definición deja al psicólogo educacional abierto a una gran heterogeneidad de funciones, que, sintetizando las señaladas por Forns (1994), Campos (1995) y Hernández (2008), consideran al menos:

a) Funciones de integración de la diversidad: que implica la detección, valoración y definición de estrategias de intervención escolar de las necesidades educativas especiales 
y de las alteraciones en el desarrollo madurativo, educativo y social de los alumnos, a fin de lograr el desarrollo de las potencialidades de todos estos.

b) Funciones de mejoramiento de la convivencia: que implica la promoción de valores como el respeto y la convivencia, y la evaluación e intervención continua de problemas de comunicación, clima, disciplina y violencia escolar.

c) Función de orientación: ligada a la orientación de los alumnos y su asesoría en la definición vocacional, y

d) Funciones pedagógico-curriculares: que incluye todas las actividades dirigidas a mejorar el acto educativo, adecuándolo al contexto, lo que implica promover y asesorar innovaciones pedagógicas; formar y guiar a directivos, profesores y apoderados, y colaborar en la elaboración de diseños curriculares y programas educativos.

Lo anterior, no obstante, debe considerar que las demandas que recibe el psicólogo también varían según el actor con el que trabaja. Así, Selvini \& Cirillo (1997) sostienen que:

a) Los docentes suelen solicitar intervenciones con fines diagnósticos y de tratamiento con alumnos que presenten problemas de adaptación o presuntos trastornos psicológicos, asesoría sobre temas psicológicos, y prestaciones como experto en relaciones interpersonales.

b) Los directivos solicitan al psicólogo trabajar en la formación de las clases, diagnóstico de éstas, intervenciones técnicas para apoyar la acción educativa, sensibilización a los docentes en la utilización de métodos de enseñanza actualizados, asesoría en innovaciones educativas, orientación escolar y vocacional de los estudiantes, e intervenciones en situaciones atípicas de los jóvenes.

c) Las familias realizan pedidos de intervenir con hijos con problemas de rendimientos o relacionales, integración de hijos con algún tipo de discapacidad, promoción de la colaboración familia-escuela, y asesoría sobre problemas educacionales y psicoeducativos.

d) A nivel de alumnos, no se observan muchas peticiones de atención, salvo cuando la presencia del psicólogo es estable al interior del establecimiento educacional.

e) Para atender adecuadamente a estas funciones y demandas, el psicólogo educacional debe actuar bajo los lineamientos de la Reforma y, por tanto, conocerla, debiendo dejar de trabajar aislado y encerrado en una oficina. De ser un clínico que está dentro de la escuela, debe pasar a integrar el sistema, mirándolo como objeto de su intervención y promoviendo el cambio al interior de éste (Banz Liendo, 2002).

\subsection{EL PSICÓLOGO EDUCACIONAL EN LA ACTUALIDAD}

Independiente de las demandas individuales de los distintos actores, las tareas específicas del psicólogo educacional en Chile son definidas por directores y sostenedores, lo que generaría una instrumentalización del rol del psicólogo en base a las necesidades administrativas y económicas del establecimiento, coartando la posibilidad de desarrollar otras áreas (Ossa, 2006).

Esta limitación se potencia con el imaginario del sistema educativo, que aún vincula al psicólogo educacional a un modelo clínico de intervención en donde se pone énfasis en los alumnos con problemas, se dirige toda la acción hacia ellos y no se permite mayor impacto en el sistema educativo (Redondo, 2007). Este patrón se evidencia en que los integrantes adultos de la comunidad escolar le atribuyen como campo de acción preferente al psicólogo la "patología del alumno", encontrándose implícita en el pedido 
de intervención la búsqueda de una solución rápida al problema que no implique excesivo involucramiento de quien ha realizado el señalamiento-usualmente el docente. El desconocimiento que los centros educativos tienen del rol del psicólogo educacional hace que éstos esperen que él solucione "mágicamente" cada problema en forma aislada a través de sus propias capacidades, sin involucrar al sistema, lo que se asocia con una visión del mismo alumno como una entidad separada, y no como parte de un sistema en el que todos los actores de la escuela se encuentran inmersos (Selvini \& Cirillo, 1997).

Lo anterior, junto con una formación deficitaria de los alumnos de psicología en el área educacional, que les impide enfrentar la complejidad de este contexto laboral (Ossa, 2006; Redondo, 2007), ha limitado y empobrecido la labor de los psicólogos en establecimientos educacionales.

Afortunadamente, la mirada de lo que pueden hacer estos profesionales se ha ido ampliando poco a poco, y si bien en la enseñanza privada -que es donde se dio inicio al trabajo del psicólogo educativo- se le restringió en un principio a una función eminentemente psicométrica, en la actualidad ha logrado ampliar su trabajo a aspectos específicamente psicoeducativos, además de estabilizar su presencia en los establecimientos educacionales particulares (Colodrón, 2004).

\subsection{EL PROBLEMA DE ESTE ESTUDIO}

Los antecedentes antes expuestos muestran que el rol que teóricamente se asigna al psicólogo educacional le abre la posibilidad de abordar las distintas dimensiones que influyen en el producto educativo y trabajar con los diversos actores que componen el sistema. Esta multidimensionalidad se contrapone con una visión más restringida de su labor, que deriva de las necesidades y recursos disponibles de quién lo emplea y de un imaginario, muy potente aún pero en progresiva retirada, que encasilla al psicólogo al espectro clínico.

A esto se suma una visión crítica de la labor del psicólogo educacional desde el estamento docente, pues percibe que los procesos llevados a cabo por él no son útiles, no se concretizan y que se habla "más de lo mismo".

Banz Liendo (2002) enfatiza que el cambio en la concepción del trabajo del psicólogo al interior de los establecimientos educativos requiere de una modificación de la cultura de estos, lo que debe ser promovido por los mismos psicólogos que trabajan en ellos. Sin embargo, para cambiar esta cultura y abrir el establecimiento a las contribuciones que el psicólogo educacional puede hacer en las distintas dimensiones de su gestión, es primero necesario conocer cuál es el rol que se le está atribuyendo en el escenario educativo real, que funciones se le asignan, que actividades éste realiza y que percepción se tiene de su desempeño. Es por este motivo que el presente estudio se propone analizar el rol del psicólogo en establecimientos particulares pagados de Concepción, considerando para esto la perspectiva de los actores que componen estos establecimientos.

\section{MÉTODO}

La presente investigación aplicó una metodología cualitativa, que se caracteriza por intentar comprender los acontecimientos desde la perspectiva de los sujetos que los 
producen y experimentan (Vieytes, 2004). Puntualmente, el trabajo se desarrolló a partir de la Teoría Fundamentada, método donde el investigador espera construir inductivamente un modelo conceptual sobre lo que los participantes ven como su realidad social (Strauss \& Corbin, 2002).

\subsection{PARTICIPANTES}

El caso en estudio está compuesto por los establecimientos particulares pagados de la intercomuna del Gran Concepción, que de acuerdo a los datos proporcionados por el MINEDUC (www.simce.cl) son 20 establecimientos que se encuentran ubicados en su totalidad en zonas urbanas y presentan un bajo índice de vulnerabilidad. De estos, el estudio incluyó a 4 establecimiento diferentes, donde se trabajó con profesores, psicólogos, directivos, apoderados y alumnos, ya que representan las diferentes perspectivas de estas comunidades educativas.

La muestra final del estudio estuvo constituida de 14 entrevistados: tres psicólogos, tres profesores, tres directivos, tres alumnos y dos apoderados. Estos fueron elegidos a partir de un muestreo teórico razonado, en donde cada uno de los estamentos se definió como un perfil de informantes (Miles \& Huberman, 1994). Desde cada perfil, los participantes fueron elegidos mediante un muestreo de máxima variación (Rodríguez, Gil \& García, 1999) y el tamaño muestral fue definido a posteriori al alcanzar la saturación teórica (Castillo \& Vásquez, 2003).

De los 4 establecimientos incluidos en el estudio, 3 de ellos contaban con psicólogo. Estos tenían entre tres meses y cinco años trabajando en el establecimiento actual. Los docentes entrevistados llevaban más de 5 años al interior de los establecimientos, siendo los tres profesores jefes, dos de ellos en nivel básico y uno en nivel de enseñanza media. Los directivos fluctuaban desde uno que se desempeñaba hace pocos meses en el cargo de rector interino, hasta otros que llevaban varios años dirigiendo el establecimiento. Los alumnos se encontraban entre $6^{\circ}$ básico y $1^{\circ}$ de enseñanza media, siendo dos de ellos mujeres, sin cargos directivos al interior de sus cursos o del colegio. Finalmente, los apoderados tenían hijos en $6^{\circ}$ y $8^{\circ}$ básico y no se encontraban desempeñando cargos directivos en el establecimiento.

\subsection{TÉCNICAS DE PRODUCCIÓN DE INFORMACIÓN}

Los datos se recolectaron a través de entrevistas semiestructuradas, que se orientaron a partir de una pauta de 6 temas: 1) funciones asignadas a los psicólogos en el establecimiento, 2) actividades que realiza el psicólogo en el establecimiento, 3) expectativas de los actores sobre el desempeño del psicólogo, 4) percepción sobre el desempeño de los psicólogos, 3) relaciones jerárquicas y de cooperación con los otros profesionales del establecimiento y 4) recursos (humanos, financieros y de infraestructura) de los que disponen los psicólogos para desempeñar su labor.

Las entrevistas fueron realizadas de manera individual por las investigadoras, en el lugar proporcionado por los establecimientos para esto y previa firma de un consentimiento informado. La totalidad de las entrevistas fueron grabadas por un equipo de audio, con la autorización del participante, y adicionalmente se registraron mediante notas de campo realizadas por los investigadores, donde se dio cuenta de lo observado durante el proceso de entrevista. 


\subsection{ANÁLISIS DE DATOS}

Para analizar los datos, se utilizó el método de comparación constante de la teoría fundamentada. En el presente estudio sólo se presentan los resultados obtenidos en la etapa de codificación Axial y Selectiva, etapa relacional del análisis de datos en donde se ligan a las categorías a través de los vínculos que emergen entre sus propiedades y dimensiones (Strauss \& Corbin, 2002).

\subsection{CRITERIOS DE RIGUROSIDAD}

En la investigación realizada, de acuerdo a la conceptualización de Castillo y Vásquez (2003), para alcanzar la credibilidad se utilizó el método de triangulación entre las fuentes, abordando distintos estamentos cuya información fue luego agrupada en categorías. Por otra parte, para lograr el criterio de transferibilidad, se realizó una descripción del contexto, lugar y características de las personas que fueron entrevistadas.

Junto a esto, y a fin de lograr el criterio de auditabilidad, se grabaron las entrevistas realizadas con un equipo de audio, las que posteriormente fueron transcritas textualmente y revisadas por los asesores. Además, se elaboró un informe de investigación exhaustivo y se efectuó el registro en un diario de campo.

\section{RESULTADOS}

A continuación se presentan los resultados del análisis relacional del estudio, exhibiendo los dos modelos conceptuales que emergieron de la codificación axial: 1) Inclusión del psicólogo y 2) Intervenciones del psicólogo con los alumnos, y el modelo teórico emergido desde la codificación selectiva: La co-construcción del rol del psicólogo.

\subsection{CODIFICACIÓN AXIAL}

En esta etapa se identificaron dos ejes de análisis: inclusión del psicólogo e intervenciones del psicólogo con alumnos. Las cuales se presentan a continuación:

\subsubsection{Inclusión del psicólogo}

Como primer fenómeno de importancia emerge la inclusión del psicólogo dentro del establecimiento educacional. La determinación de incluirlo se comprende como derivada, por un lado, de problemas conductuales en los alumnos del establecimiento, que son detectados por profesores, directivos y orientadores, y por otra parte, del asumir, como equipo de trabajo, una limitación de la comunidad educativa para enfrentar dicha problemática, en la que se hace evidente una falta de herramientas en los profesionales del establecimiento y/o una falta de profesionales idóneos para enfrentar este tipo de problemáticas.

"Mira, nosotros veíamos que habían muchas problemáticas de los chiquillos que los orientadores no podían solucionar, de repente muchas situaciones que las profesoras no sabían como enfrentarlas, o los orientadores se sentían como limitados, entonces decidimos, hace ya algunos años atrás contratar a una psicóloga..." (Directivo 3). 
Esto, en conjunto con el modelo de otros colegios que ya cuentan con un psicólogo dentro del establecimiento y que funcionan como ejemplos a seguir, lleva a que el equipo directivo tome la decisión de incluir a un psicólogo dentro de su equipo.

Dentro de las condiciones que se encuentran influyendo en la forma en que se presenta el fenómeno de la inclusión del psicólogo en el establecimiento educacional y, específicamente, si esta inclusión es en jornada completa o media jornada, se encuentra la intensidad de la necesidad percibida y la evaluación del trabajo realizado por el psicólogo, ya que en ocasiones ingresa al colegio con media jornada de trabajo y al poco tiempo, tras una evaluación positiva de su desempeño, es contratado en jornada completa.

"Mira, yo llegué aquí como ex alumna a ofrecer gratis algunos trabajos y presenté unos programas de prevención... entonces, a fines de ese año me llamaron a mí para decirme que ellos querían contar con mi presencia, pero ya no en forma voluntaria y esporádica, sino que en forma sistemática y permanente y me ofrecían un trabajo..." (Psicólogo 3).

Al comienzo, cuando el psicólogo recién llega a trabajar al establecimiento, se produce una confusión con el rol que viene a desempeñar, ya que muchas veces se piensa que viene a ejercer un rol clínico. Esto influye en la forma en que el psicólogo se incluye dentro del establecimiento, dado que debe aclarar estas dudas, progresivamente ir poniendo límites y focalizando las expectativas de la comunidad educativa, lo que, de acuerdo a lo que manifiestan los psicólogos, es una labor desgastante. A pesar de esto, es importante destacar que el psicólogo siempre es bien recibido por los miembros del establecimiento, quienes se encuentran dispuestos a integrarlo y apoyarlo en sus funciones, lo que refleja que el desgaste y la confusión que sienten los psicólogos, sólo es sentido por ellos y no por el resto de la comunidad educativa.

"Al principio es súper complicado, porque hay confusión respecto a lo que uno viene a hacer, tuve que poner muchos límites en lo que yo venía a hacer y lo que no me correspondía, y eso igual es súper desgastante para uno..." (Psicólogo 4).

Una vez que el psicólogo es incluido en el establecimiento, éste realiza actividades, especialmente con los alumnos, con la finalidad de resolver las demandas iniciales de estos, esto es, los problemas percibidos por la comunidad educativa y que inicialmente llevaron a su contratación. Además, frente a su mera llegada los miembros de la comunidad educativa se muestran esperanzados en que el psicólogo podrá resolver las dificultades que no han podido resolver solos.

Todo el proceso anterior, se entiende como parte del nuevo escenario que genera la Reforma Educacional, que le otorga nuevas funciones al sistema educativo y donde ya no sólo debe preocuparse del proceso de enseñanza aprendizaje, sino del alumno en su totalidad. Otro aspecto importante a considerar dentro del contexto en el cual se determina la inclusión del psicólogo en el colegio, es el progresivo aumento y la mayor importancia que se le otorga socialmente a los problemas conductuales y la violencia escolar presentados por los estudiantes dentro de sus establecimientos. El enfrentarlos, y dar respuesta a la preocupación social respecto a estas temáticas, lleva a que los establecimientos incluyan al psicólogo en la comunidad educativa. 


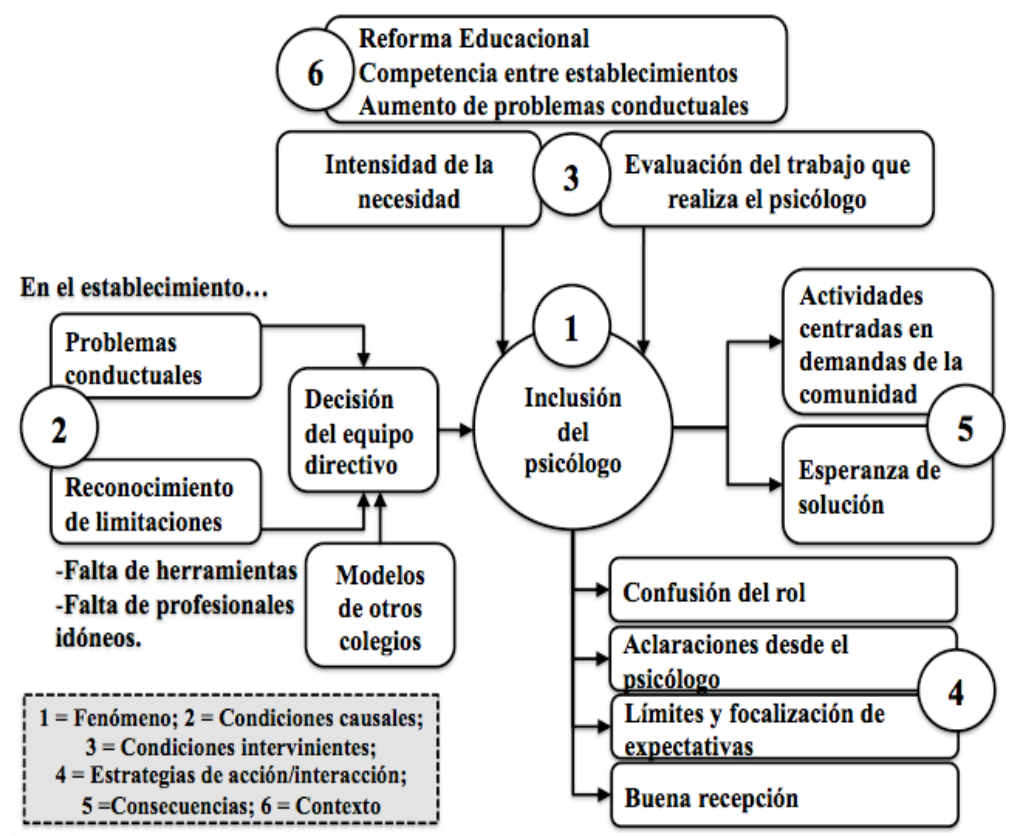

Figura 1. Modelo conceptual de la codificación axial: Inclusión del psicólogo

\subsubsection{INTERVENCIONES DEL PSICÓLOGO CON ALUMNOS}

Como segundo fenómeno de importancia emergieron las intervenciones que el psicólogo realiza dentro del establecimiento con los alumnos, que pueden llevarse a cabo a nivel individual o grupal.

Dichas intervenciones derivan de la detección realizada por el psicólogo de problemas conductuales, personales o a nivel de curso, o de peticiones realizadas por profesores, directivos, orientadores o la Unidad Técnico Pedagógica, que también se sustentarían en la observación del estudiantado. En ocasiones, esto permite incluirlas dentro de planificaciones anuales o semestrales del psicólogo, aunque en otros casos la intensidad o novedad de un problema hacen que la intervención deba realizarse de forma emergente.

"Las funciones se las va describiendo en terreno directo el campo de orientación, y en el contexto general la unidad técnico pedagógica, que es la entidad que supervigila y que está sobre ellos como unidad de orientación. Entonces la psicóloga se ve enfrentada en terreno a la propuesta que le haga el profesor de asignatura, el profesor de jefatura, la orientadora del nivel..." (Directivo 2).

Por otra parte, dentro de las condiciones que influyen en que las actividades del psicólogo sean individuales o grupales, se encuentra por un lado la experticia del psicólogo en ciertos temas, que lo llevan a proponer intervenciones centradas en aquellas problemáticas en las cuales se considera más experto y en la evaluación que éste hace de cual sería la estrategia más adecuada. Esta estrategia también considera la apreciación del 
psicólogo u otro profesional sobre la causa del problema, ya sea esta personal-familiar, conductual o alguna problemática a nivel de curso, ya que esto también aportará en la definición de la modalidad individual o grupal de la intervención.

Así, la intervención que realiza finalmente el psicólogo es definida por él, aunque los otros miembros de la comunidad educativa pueden aportarle antecedentes o indicarle sobre qué temas intervenir.

“...hay otras cosas que pide el rector, los profesores, el vicerrector, etc., pero en general todas las intervenciones grupales se me han ocurrido a mí, sólo me han dicho con quién trabajar, pero yo veo qué hacer" (Psicólogo 4).

Los diferentes actores de la comunidad educativa reaccionan de manera positiva a las intervenciones que realiza el psicólogo dentro del establecimiento, las evalúan positivamente y le facilitan los recursos que él requiera para éstas, ya sean materiales, infraestructura, personal o permitiéndole a los alumnos asistir a las actividades programadas por el psicólogo.

"Bueno, él dispone del equipo de profesores, dispone de la dirección, dispone de una oficina, dispone de todo el recurso tecnológico para realizar bien su tarea, dispone de los alumnos en el momento que es necesario que ella converse con los alumnos" (Directivo 3).

No obstante, es necesario distinguir que el acceso a los recursos materiales y de infraestructura se le otorga sólo por el hecho de trabajar en el establecimiento, mientras que el acceso a los alumnos y la colaboración de otros profesionales aparece como la consecuencia de la evaluación positiva que hacen de su quehacer.

Las consecuencias que se atribuyen a intervenciones del psicólogo, se observan principalmente en la mejora de la capacidad de resolución de conflictos de los alumnos y en cambios conductuales, especialmente en aquellos estudiantes que presentaban más dificultades al momento de la inclusión del psicólogo en el establecimiento. Cabe señalar, no obstante, que sólo los psicólogos parecen capaces de aludir a resultados específicos, mientras que los otros actores muestran más bien una apreciación global.

“...he visto cambios conductuales, más cambios especialmente en los que tenían más problemas...” (Psicólogo 4).

“...en la resolución de conflictos, se han visto importantes logros...” (Psicólogo 3).

Dentro del contexto en el cual se insertan las actividades realizadas por el psicólogo con los alumnos, se encuentra el aumento de problemas conductuales de los estudiantes y de bullying a nivel nacional. Lo anterior, incluso, ha llevado al Ministerio de Educación a tomar medidas al respecto, ante lo cual planteó un plan de políticas de convivencia escolar para enfrentar estas problemáticas y apoyar la formación de valores de convivencia entre los alumnos, sentando lineamientos que el psicólogo debe considerar. 


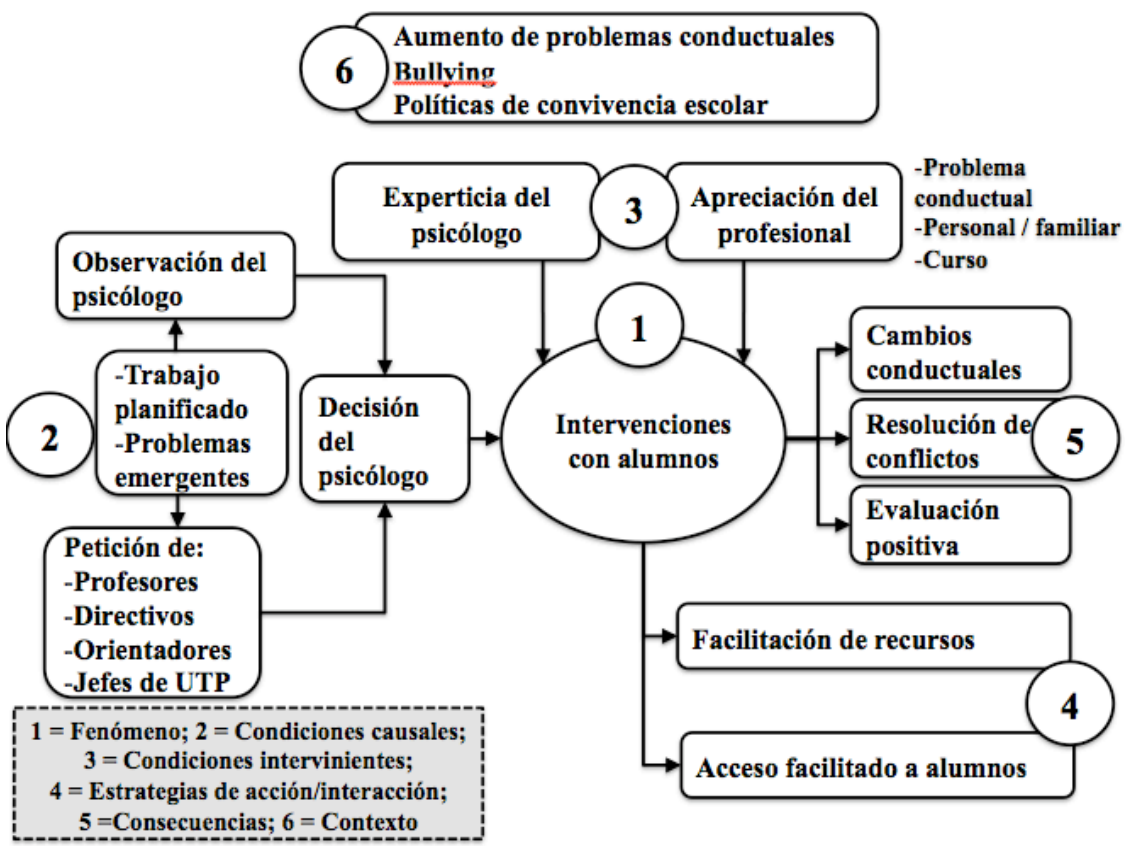

Figura 2. Modelo conceptual de la codificación axial: Intervenciones del psicólogo con alumnos

\subsection{CODIFICACIÓN SELECTIVA}

Como fenómeno central de la investigación, emergió el proceso de co-construcción del rol del psicólogo que trabaja en un establecimiento educacional particular.

Dicho proceso de co-construcción del rol es realizada en conjunto por el psicólogo y los otros miembros de la comunidad educativa, y se inicia con la inclusión del psicólogo, lo que es desencadenado por la constatación de problemas en los alumnos, generalmente a nivel conductual y, junto a lo anterior, la percepción que la comunidad educativa no puede enfrentarlos con los recursos que cuenta. Estas dificultades van a determinar en cierta medida el rol que va a tener el psicólogo en el colegio, ya que, al menos en una primera instancia, este profesional deberá enfocarse en dar solución a dicha problemática.

Esta focalización en la demanda también deriva de que la comunidad educativa no identifica otras opciones pues no tiene una representación previa de lo que el psicólogo puede hacer. De esta forma, el psicólogo no puede orientarse a otras tareas, dada la falta de claridad existente en el colegio respecto al rol que puede desempeñar y a la falta de conciencia de ellos mismos del aporte que pueden generar. Es así como los mismos psicólogos reconocen no saber específicamente qué es lo que deben hacer en el colegio.

“...como no hay claridad al respecto uno tiene que ir adecuando su rol dentro del colegio a lo que a uno le piden y también según lo que tu aprendiste y sabes...” (Psicólogo 4). 
Uno de los factores que se encuentran influyendo en esta falta de claridad es que los colegios que lo han incluido, es la primera vez que cuentan con este profesional dentro del establecimiento, por lo tanto, no poseen un modelo previo sobre lo que el psicólogo debería desarrollar dentro de la institución, lo cual a su vez, lleva a que se inicie la construcción del rol dentro del establecimiento recién con la llegada del psicólogo.

De esta forma, el psicólogo va a decidir en una primera instancia lo que tiene que hacer dentro del establecimiento de acuerdo a la demanda inicial definida por el centro educativo. Esta decisión es producto de los intereses o experticia que posee este profesional, de la autopercepción de sus capacidades y de los lineamientos generales del colegio sobre las temáticas en las cuales el psicólogo debe focalizar su trabajo. Estos tres aspectos llevan al psicólogo a determinar el tipo de actividades que realizará, pues la comunidad educativa sólo apunta el problema que debe enfrentar, pero no se pronuncia en torno a la forma de abordarlo.

“...en general todas las intervenciones grupales se me han ocurrido a mí, sólo me han dicho con quién trabajar, pero yo veo qué hacer" (Psicólogo 4).

Estas primeras actividades realizadas por el psicólogo, van a ir generando una noción inicial de lo que el psicólogo puede hacer y de lo que se puede esperar de él. Sin embargo, se observa que no sólo el psicólogo influye en la construcción de su rol, sino que también lo hará el establecimiento educacional, especialmente a través de sus expectativas difusas respecto a los problemas que el psicólogo puede abordar y de las competencias que debería poseer. Estas expectativas sumadas a la autopercepción de insuficiencia de recursos profesionales en el resto de la comunidad educativa para enfrentar los problemas detectados, llevan a considerar al psicólogo como la solución. El psicólogo es visto en ese momento como un profesional que podrá dar solución a todos los problemas del establecimiento.

"Es súper complicado cuando uno llega a un colegio, porque llega el psicólogo y creen que tú vas a salvar el mundo que tú vas a solucionar todo..." (Psicólogo 4).

Todo lo anterior produce un ciclo, en el cual las actividades realizadas por el psicólogo derivan de los factores antes mencionados: el problema definido por el establecimiento, las expectativas de éste, un rol poco claro fruto de la falta de experiencia con psicólogos, las competencias e intereses del profesional, y la libertad que se le da para definir un plan de acción. Las actividades que este finalmente realiza, van generando nuevas expectativas y consolidando y perfilando las anteriores, lo que a su vez influye en la definición de las actividades futuras, tanto las solicitadas al psicólogo como las decididas por él. Es esta retroalimentación entre la generación de actividades y expectativas la que lleva finalmente a la construcción del rol basado en las experiencias que se van generando desde su inclusión.

Los resultados de las actividades realizadas por el psicólogo son bien evaluados por los otros miembros de la comunidad educativa, dada la falta de una imagen previa del rol y una consecuente baja capacidad crítica respecto del mismo.

"Es que yo estoy encantada con ella ya, o sea me encanta la labor que hace, me encanta la forma en que ella llega, me encanta la forma en que ella se plantea..." (Profesor 2). 
Ante esta evaluación que tiende a ser positiva, el psicólogo continúa llevando a cabo las mismas actividades, lo que conlleva una atrofia e hipertrofia de su rol, ya que continúa realizando estas actividades y no desarrolla nuevas intervenciones.

Todo este proceso de co-construcción del rol del psicólogo en el sistema educativo, se da dentro de un contexto determinado por la Reforma Educacional que rige en nuestro país, que ha llevado a otorgar otras responsabilidades al sistema educativo, quién ya no sólo se debe preocupar del aprendizaje del alumno, sino también de su desarrollo integral. Tal situación lleva a que el psicólogo realice actividades dentro del establecimiento en pos de este objetivo y de esta manera se va determinando la construcción del rol.

Otro aspecto también importante a considerar dentro del contexto, es la competencia que se da actualmente entre los establecimientos, especialmente a nivel de los establecimientos particulares pagados, quienes luchan por tener mejores resultados y atraer alumnos. Esta lucha entre los colegios, lleva en muchas ocasiones a determinar la inclusión y funciones que deberá desempeñar el psicólogo dentro del establecimiento, ya que si uno de estos establecimientos incluye a este profesional, otros colegios seguirán su ejemplo tanto en la inclusión como en las funciones que se le solicitarán, principalmente motivados por la valoración social que esto significa.

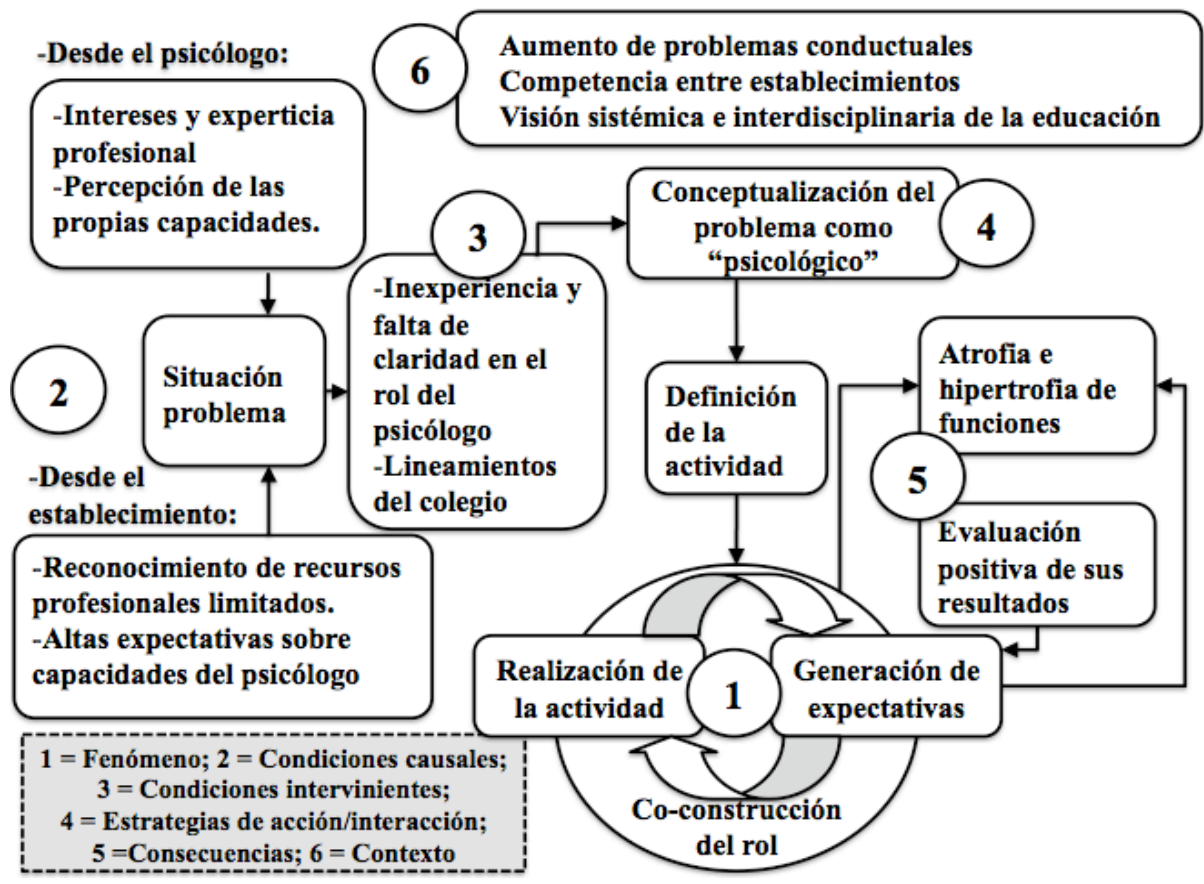

Figura 3. Modelo conceptual de la codificación selectiva: Co-construcción del rol del psicólogo 


\section{DISCUSIÓN}

A partir de la década del 90 se han producido en el país diversos cambios sociales, culturales y tecnológicos que han generado nuevas demandas y transformaciones en el sistema educativo. Una de estas demandas, que la comunidad educativa ha hecho especialmente a los docentes, dice relación con una mayor responsabilidad de éstos en la formación integral de los estudiantes, más allá del plano netamente académico.

A partir de las entrevistas realizadas en este estudio, se desprende que estas nuevas responsabilidades han llevado, paulatinamente, a incorporar en los establecimientos educacionales a otros profesionales, y a la creación de equipos multidisciplinarios de trabajo como herramienta para enfrentar estos nuevos desafíos. Sin embargo, estos equipos, conformados principalmente por orientadores y psicopedagogos, han ido detectando falencias en su capacidad de enfrentar situaciones que no correspondían a sus áreas circunscritas de trabajo, especialmente problemáticas a nivel conductual en los estudiantes.

Es por estas razones que, especialmente en los colegios particulares pagados, y debido a los mayores recursos disponibles y la libertad de gestión, se han ido incluyendo psicólogos en los equipos, para suplir las falencias sentidas y apoyar, especialmente al docente, en los nuevos desafíos que la reforma y el cambio sociocultural le han impuesto.

Ahora bien, la literatura respecto a la función que debe desempeñar el psicólogo al interior del establecimiento educacional es amplia y diversa, no existiendo un total acuerdo entre expertos, respecto a la labor especializada que este profesional deba desempeñar. Esta escasa delimitación del rol del psicólogo se repite en la realidad de los establecimientos educacionales, donde se evidencia una confusión en torno a las funciones que viene a desempeñar el psicólogo dentro del colegio.

Puesto que no hay claridad, se observó que este rol se va construyendo entre el psicólogo y la propia comunidad educativa. Se trata de una co-construcción de su rol, en la que se ponderan las necesidades particulares del sistema educativo en el cual se encuentra inserto, y las competencias e intereses que este profesional posee, lo cual determina la oferta que este hace y finalmente las intervenciones que realiza. Debido a esto, y a la falta de experiencia previa con psicólogos dentro del establecimiento que estipulen pautas sobre el accionar de este profesional, es que las intervenciones del psicólogo van a ser los fenómenos sobre los cuales los otros miembros de la comunidad educativa sustentarán su construcción de este rol.

Los otros miembros de la comunidad educativa, al momento de la inclusión del psicólogo en ella, presentan altas expectativas respecto a las competencias y a las actividades que este profesional realizará dentro del establecimiento. Es por esto que los entrevistados en este estudio, en conformidad a lo planteado por Selvini \& Cirillo (1997), esperan que él le dé solución a todos los problemas sentidos por la comunidad, lo cual también viene a determinar, al menos en una primera instancia, las actividades que el psicólogo realiza, y con esto, determinar también el rol del profesional dentro del establecimiento.

La falta de claridad no sólo se observa dentro de los otros miembros de la comunidad educativa, también se constata a nivel de los psicólogos. Cabe destacar en este aspecto que los psicólogos entrevistados reconocen una falta de definición del rol, no obstante, ellos perciben que pueden cumplir con lo que los establecimientos les demandan. Esta falta de definición del rol reconocida por los psicólogos pudiera encontrar sustento en lo que Ossa (2006) y Redondo (2007) plantean en relación a la formación en el área 
educacional a nivel de pregrado en las distintas carreras de psicología, la que sería deficiente para desempeñarse en contextos educativos.

Finalmente, vale decir que la falta de claridad respecto al rol no es necesariamente un aspecto negativo, o perjudicial para el desempeño del psicólogo dentro del establecimiento educativo, ya que puede brindar mayor flexibilidad para que este profesional tome en consideración el contexto general en el cual se encuentra el establecimiento, y pueda aportar intervenciones específicas que mejoren el funcionamiento general del sistema, lo que a su vez lleva a mejorar la calidad de la educación otorgada.

La importancia de la consideración del contexto específico del establecimiento y las repercusiones que esto tiene en la calidad de la educación, es manifestada por autores como Banz Liendo (2002) y Diversidad Educativa (2007) entre otros, quienes recalcan que es primordial crear una intervención específica para cada establecimiento, considerando sus características particulares, tanto positivas como negativas. Ahora bien, esta necesidad de considerar el contexto personal, educacional y familiar en las intervenciones realizadas por el psicólogo, es manifestada por los entrevistados y es parte de los productos positivos que se obtienen de la co-construcción.

A partir de los resultados encontrados en el presente estudio, también es importante destacar que el psicólogo considera que cuenta con todos los recursos que necesita a nivel humano, financiero y de infraestructura, que le permiten realizar de manera adecuada sus funciones. No obstante, también establece que él morigera sus requerimientos según el contexto en el que se desempeña.

En referencia a las peticiones de trabajo que recibe el psicólogo en el contexto escolar, se observa que éstas provienen de los diversos miembros de la comunidad educativa. Al respecto, Selvini \& Cirillo (1997) realizan una distinción de estas peticiones en base a quien las solicita, distinguiendo entre las realizadas por los docentes, directivos, familias y alumnos.

En concordancia con estos autores, los datos obtenidos en la presente investigación, especialmente en relación a las peticiones realizadas por los docentes, familia y alumnos, evidencian que los docentes, en su mayoría, solicitan al psicólogo apoyo clínico para los alumnos; la familia busca apoyo para las dificultades académicas e interpersonales de sus hijos y se aprecian escasas peticiones nacidas directamente por parte de los alumnos.

Estos mismos autores plantean que los directivos esperan que el psicólogo se involucre en el currículo académico y en su implementación. No obstante, ésta no es una petición realizada en el contexto estudiado, principalmente debido a que no existe el espacio de acción para que el psicólogo intervenga en esta área. Según Forns (1994), esta sería una de las funciones asignadas más recientemente al psicólogo, por lo cual podemos hipotetizar que esto aún no se ha incorporado a la realidad del contexto investigado.

Es importante destacar, como reflexión final, que no es fundamental establecer un único rol para los psicólogos que se encuentren trabajando dentro de establecimientos educacionales sino, más bien, considerar el contexto como un aspecto principal en la determinación de las funciones que el psicólogo deberá desempeñar. Determinar un único rol para todos los psicólogos, coartaría el accionar y la posibilidad de que su labor sea realmente significativa para el establecimiento.

Frente a esto, son los mismos psicólogos y aquellos profesionales vinculados a la educación quienes deben dejar de buscar una pauta de acción que determine las intervenciones a realizar por parte del psicólogo dentro del sistema educativo, y abrirse a 
la experiencia de ir juntos descubriendo las intervenciones realmente apropiadas para el contexto específico de cada establecimiento educacional, dando así solución a los requerimientos y necesidades propias de cada institución.

Lo anterior no desconoce, no obstante, que la falta de información de los psicólogos y de la comunidad educativa sobre el rol que puede jugar este profesional puede terminar limitando el aporte de éste último y encerrándolo en rutinas estereotipadas y de impacto reducido. Sin embargo, parece que más importante que delimitar un rol único para el psicólogo que se desempeña al interior de los establecimientos educacionales, este estudio nos lleva a destacar que es primordial que, en la práctica, cada establecimiento se auto observe, y a partir de ello determine las funciones que requerirá por parte del psicólogo.

En relación a las limitaciones del presente estudio, podemos destacar que este sólo responde al contexto de los colegios particulares pagados del gran Concepción, por lo que los resultados obtenidos podrían no ser replicables en establecimientos educativos con características distintas en cuanto a nivel sociocultural y económico, ya que lo principal a considerar respecto al rol del psicólogo al interior del centro educativo, es su contexto particular y sus necesidades específicas.

Finalmente, a partir de los resultados obtenidos en esta investigación, y de las conclusiones derivadas de ésta, surge la necesidad de realizar estudios similares en establecimientos con realidades administrativas y socioeconómicas diferentes, ya sea de tipo particular subvencionado o municipalizado, para conocer los alcances del rol del psicólogo en este tipo de establecimientos. Además, y de forma posterior a la realización de estos estudios, resultaría de gran utilidad realizar un estudio comparativo con respecto al rol del psicólogo en los distintos tipos de centros educativos, para rescatar sus puntos de concordancia y de discrepancia.

\section{REFERENCIAS BIBLIOGRÁFICAS}

American Psychological Association [APA], Commission for the Recognition of Specialties and Proficiencies in Professional Psychology (2005). Archival Description of School Psychology. Consultado en Septiembre 22, 2008 en http://www.apa.org/crsppp/schpsych.html

Arancibia, V., Herrera, P. \& Strasser, K. (1997). Manual de Psicología Educacional. Santiago: Pontificia Universidad Católica de Chile.

Banz Liendo, C. (2002). El rol del Psicólogo Educacional en tiempos de Reforma: Desde el clínico en la escuela al mediador de la institución. Consultado en Octubre 23, 2008 en http://www. ecampus.cl/Textos/psicologia/Cecilia_Banz/cbanz.htm.

Barca, A. (1998). Relaciones y aportaciones de la psicología a la educación. Papeles del Psicólogo, 71. Consultado en Octubre 2, 2008 en http://www.papelesdelpsicologo.es/vernumero.asp?id=792.

Campos, F. (1995). El rol del psicólogo de la educación. Papeles del psicólogo, 63. Consultado en Agosto 8, 2009 en http://www.papelesdelpsicologo.es/vernumero.asp?ID=690

Castillo, E. \& VÁSQUEZ, M. (2003). El rigor metodológico en la investigación cualitativa. Colombia Médica, 34 (3), 164-167.

Colodrón, M. (2004). Rol del psicólogo en la educación. Colegio Oficial de Psicólogos de España. Consultado en Octubre 19, 2009 en http://www.cop.es/colegiados/M-02744

Diversidad Educativa (2007) Los psicólogos y el futuro de nuestra educación: Entrevista a Rodrigo Cornejo. Consultado en Agosto 8, 2009 en http://diversidadeducativa.cl/LOS\%20 PSICOLOGOS $\% 20$ Y\%20EL \%20FUTURO $\% 20$ DE\%20NUESTRA\%20EDUCACIÓN_\%20 ENTREVISTA\%20A\%20RODRIGO\%20CORNEJO.pdf 
Eyzaguirre, B. \& Le Foulon, C. (2001). La calidad de la educación chilena en cifras. Estudios Públicos, 84, 85-204.

Forns, M. (1994) El psicólogo en el contexto educativo. Anuario de Psicología, 63, 187-211.

Hernández, P. (23 de enero de 2008). Los campos de acción del psicólogo educativo. Psicologíacientífica.com. Consultado en Octubre 28, 2008 en http://www.psicologiacientifica. com/bv/psicologia-321-1-los-campos-de-accion-del-psicologo-educativo.html.

Mena, I. (1992). ¿Qué es la psicología educacional? Apunte de clase curso Psicología Educacional, Escuela de Psicología Pontificia Universidad Católica de Chile. Documento no publicado.

Miles, M. \& Huberman, A. (1994). Qualitative data analysis: an expanded sourcebook. California: Sage.

Observatorio Chileno De Políticas Educativas, OPECH (2006). ¿Por qué hay que cambiar la LOCE y revisar la municipalización? Consultado en Agosto 25, 2009 en http://www.opech. cl/Libros/doc5.pdf

Ossa, C. (2006). Factores que estructuran el rol del psicólogo en educación especial. Revista de Psicología de la Universidad de Chile, 15 (2), 131-146.

Raczynski, D. Y Muñoz, G. (2007). Reforma Educacional Chilena: el difícil equilibrio entre la macro y la micropolítica. Revista Iberoamericana sobre Calidad, Eficacia y Cambio en Educación, 5, 3.

Redondo, J. (2007). Aportes del Psicólogo Educacional a los Establecimientos Educativos. Consultado en Octubre 20, 2009 en http://www.opech.cl/bibliografico/calidad_equidad/ aporte_psi_educ_centros_educativos.pdf

Rodríguez, G., Gil, J. \& García, E. (1999). Metodología de la investigación cualitativa. Málaga: Aljibe.

Santander, M. (2006). Ideas para una educación de calidad. Santiago: Libertad y Desarrollo.

Selvini, M. \& Cirillo, S. (1997). El mago sin magia. Argentina: Paidós.

Strauss, A. \& Corbin, J. (2002). Bases de la investigación cualitativa. Técnicas y procedimientos para desarrollar la teoría fundamentada. (2a. ed.). Bogotá: Universidad de Antioquía.

Vieytes, R. (2004). Metodología de la investigación en organizaciones, mercado y sociedad: Epistemología y técnicas. Buenos Aires: De las ciencias.

Woolfolk, A. (2006). Psicología Educativa. México: Pearson Educación. 